\title{
Paradigm Control of Complex Technical Systems
}

\author{
Sergey V.Bublichenko \\ Volodymyr Dahl East-Ukrainian National University, Ukraine
}

Copyright (C) 2015 Horizon Research Publishing All rights reserved.

\begin{abstract}
The problems associated with the concept of extreme principle, structural decomposition, entropy and the possibility of using the methods of the theory of categories. The most common results of statements of problems and mathematical models of management in the structure of the cutting technology the semiconductor ingots and other functional materials for thin plates for the preparation of base layers for the micro - and nanoelectronic devices are presented.
\end{abstract}

Keywords Control, Management, Paradigm, Complex System, Technical System, The Theory of Categories and Functors, Entropy, Extremum Principle

\section{Introduction}

One of the major problems the solution of which depends on the efficiency of the synthesis of control of modern technological systems is the building an effective a mathematical model capable to influence the optimization parameters. Thus the basic process of constructing this model is to find out how the resulting model is adequate to reality. As you know, the lack of sufficient statistical information about the functioning of technological systems, the need to incorporate in the model a huge number of internal connections between the elements of real systems is often (when using deterministic mathematics) to unjustified idealization level of technological operations and processes. Thus model produced by traditional, do not qualify for checking their compliance with the simulated system. However, for maximum flexibility of technological systems, these systems must meet certain other requirements of system integrators.

In connection with the foregoing, the problem of synthesis of control of technological systems at a substantiation of design decisions may qualify as sufficiently topical.

In the more general case where the number of possible problems, or very large, or at all foreseen in advance, the existing methods of system analysis does not give clear and precise answers to questions about the potential sources of technological systems universality, and the problem of their synthesis becomes difficult to solve. The reason - the lack of conceptual models of system-wide universal systems suitable for different technologies, sometimes very distant from each other, which is a clear mezhdistsiplinarnist task, making it difficult to find a suitable formalism. Finally, there is confusion in most conditions, the degree of universality of various technological systems - as a consequence, the absence of the same general theoretical interdisciplinary framework.

While by scientists, in particular Ludwig von Bertalanffy [1], M.D.Mesarovic and Y.Takahara [2] et al. repeatedly expressed between the disciplinary apparatus of the theory of system research and related concepts. However, serious attempts to extend and adapt these formal concepts relative to the maximum of a large class of real objects technologies, and study the features thus obtained formal models in terms of their potential completeness and versatility still almost was not. All known attempts are generally reduced to facts or algorithmization many, if not all, of the physical processes around us, or to the use of algorithmic complexity theory of information objects to prove constructive precisely such processes and their results.

On the other hand, issues related to the concept of a universal systems and universality in general are extremely complex. They anyhow comprise a wide range of related problems. One of them - the problem of the general applicability of mathematics and its methods. Indeed, the universality of the mathematical apparatus acknowledged. However, the reasons for this universality still argue and mathematicians, and philosophers, and specialists in other fields. The theory of algorithms and recursive functions, the Turing-Church thesis to some extent explain the purpose computing device mathematics but does clarify the situation regarding the causes of the universal applicability of mathematical formulas and relations in various fields of human activity - from sociology and genetics to astrophysics and quantum physics. Therefore, exploring the notion of a universal system - in information processing technology or engineering technology, nanotechnology, we will inevitably have to deal with the general mathematical and general philosophical problems associated with the criteria of universality, with their possible formal relations for various technologies used both human and nature. During this type of analysis and the question naturally arises about the management of technological systems considered as formal 
models. As already mentioned, it is necessary that such models are universal and cross-cutting (mechatronic), i.e. applicable in principle to all technologies and systems - from mathematics to biology, or apply the criteria of universality and the position of the various technologies on the overall scale of their potential will remain unsolved. Thus, the actual problem is the development of interdisciplinary theory for describing models of universal systems in general, and this theory must be mathematical, or at least such that preserves the basic logical structure of modern mathematics and its universal evidentiary apparatus.

Objective of the work is to create a conceptual theoretical framework and appropriate interdisciplinary formal mathematical apparatus to describe the system analysis, simulation and synthesis of control systems engineering versatile technology, improve efficiency and quality of the technological process of production of microelectronic substrates and nanoelectronic devices by optimization of machining processes of semiconductor and other functional materials based on the development of a methodology for constructing fuzzy models of production technology and management in the neural.

\section{Materials and Methods}

\subsection{Basic Level Representation of the Studied System}

We shall stipulate the use of terms. The following sections will be sequentially introduced concepts such as the structure of the system and the structure of the invariant - the image of a particular representation of a set of systems in numerical sets. Thus, the information contained in the system to understand the structure of the system; invariant structure will be called the number of such information; of conformity that can show system - morphisms.

Replace the concept of "information" is the concept of "structure", which itself is very polymorphic and requires clear explication. Before you do by it, let us consider a few examples from the practice of describing structures of complex systems [3]. Thus, the technological process of cutting circuits, wherein different circuits according to the needs of continuous resource (it may be divided into groups of influence of deformation of the cutting edge changes, the tension of sharpening, the cutting force). Convenient to describe such a technological process with the set of elements of $\boldsymbol{n}$ (schemes), divided into disjoint classes $\boldsymbol{w}$ (by type of movements and types of provisions of the cutting tool and monocrystal), with the number of elements in the class $n_{i}\left(i=\overline{1, w} ; \quad \sum_{i=1}^{w} n_{i}=n\right)$, in other words, the mathematical structure of the partitioned sets.

Under the structure we mean the mathematical structure, as it is understood, for example, in [3-5]. Convenient language for formalizing the notion of "mathematical structure" is the language of category theory $[6,7]$. And in the following wording under the technical structure of the system will understand the structure of the object of this category which describes the system, for example, a structure with a specific set of relationships, the structure of a particular technical system or structure of fixed topological space $[2,7]$.

Thus, the system is to explicate the category whose objects are to be interpreted as the state of the system. The essential feature of the categorical description of systems is the need to specify not only the possible states of the system, but also the permissible methods of transforming one state to the other - morphisms. Moreover, if the category of object classes are the same, and the morphisms are different, then, strictly speaking, these categories represent the different systems. Sam look at the system as a category quite justified $[2,6,7]$. By specifying a system of categories, we fix the structure unambiguously include in the category of objects can exist only "on view" different from each other, but have the same structure, for example, the objects into each other at the change in the index from the elements. Among the categories can meet and indistinguishable from each other morphisms, for example, through what - either the symmetry properties of the displayed items. Thus, a given category axiomatic its objects (the ratio, the topology, the laws of the composition, etc.) and a set of admissible morphisms, we fix a "provisional structure" of the system. It should learn to compare the various states of the systems and methods of the transition between them, and the like, and to distinguish dissimilar structure. In other words, it is convenient to have the order relation and equivalence relation on explication system category. The usual way of comparing the mathematical structures - a comparison using the homomorphism: declares that the structure of the object $\boldsymbol{A}$ weaker object structure $\boldsymbol{B}$, if there is a certain kind of homomorphism structure of the $\boldsymbol{A}$ in the $\boldsymbol{B}$; If said homomorphism exists as of $\boldsymbol{A}$ in $\boldsymbol{B}$, and from $\boldsymbol{B}$ to $\boldsymbol{A}$, the structure of the $\boldsymbol{A}$ and $\boldsymbol{B}$ are the same. For example, the cardinality of the set of $\boldsymbol{A}$ less power $\boldsymbol{B}$, if there is an injection of $\boldsymbol{A}$ in the $\boldsymbol{B}$, if there are as injection of $\boldsymbol{A}$ in $\boldsymbol{B}$ and $\boldsymbol{B}$ injection of a $\boldsymbol{A}$, then the principle of trichotomy there is a bijection between the $\boldsymbol{A}$ and $\boldsymbol{B}$, and these sets are equicardinal. Cardinality of the set is a typical example of the proposed design structures (preliminary structure - the category of sets with correspondences as a morphism, a special homomorphism - monomorphism this category, or injection, the structure - the power, or the cardinal number of sets). It is this principle that underlies the design of the comparison structure used in this work.

Another remark. From the point of view of algebra arbitrary category is a partial semigroup, so natural to introduce an equivalence relation on the category through the factorization for some of its subcategories. Moreover, that the analogy with groups [5] does not exclude the fact that the equivalence relation compatible with composition in the semigroup is reduced to factorization on a "normal" categories.

There is another mathematical structure which can serve 
as a tool for comparing the structures. If the homomorphism binds "same type" structure - in this case, the objects of a category, then presentation of one category to the other allows you to compare the structure generated by different axiomatic. In particular by means of representations we can explore unfamiliar objects of nature by means of analytically well-designed structures. For example, the physical objects with different types of symmetries described in the representations of the group of matrices. The same methodological technique - representation in the structure of the investigated structures are well-known sets of numbers is the basis of all quantitative description of systems and phenomena. It is proposed to use views to reduce the comparison of arbitrary structures to streamline the cardinal numbers and, more importantly, to specify conditions that are sufficient for this purpose. This possibility is inherent in the design categories. The fact is that, by definition, an arbitrary category, each pair of its objects $(\boldsymbol{A}, \boldsymbol{B})$ is mapped object of the category of sets - the set of morphisms $\operatorname{Hom}(\boldsymbol{A}, \boldsymbol{B})$ and power sets, thus can be a versatile tool comparison of categorical structures.

The main result of the study of representations of arbitrary categories cardinal structure of sets is as follows. Power CardHom $(\boldsymbol{A}, \boldsymbol{B})$ Nom sets $(\boldsymbol{A}, \boldsymbol{B})$ of morphisms from $\boldsymbol{A}$ to $\boldsymbol{B}$ - is the same for objects with matching structure (so called invariant structure), as for the number of distinct structures CardHom $(\boldsymbol{A}, \boldsymbol{B})$ are arranged in the same way as the structures themselves: more structure to the larger number. For consistency, streamlining structures and numbers enough to homomorphism, with which we compare the structure of objects in a certain way correlated with the monomorphism category. Before proceeding to the solution of the existence of invariants in structures, give a somewhat less specialized definition of invariants that help to understand the categorical terminology invariant CardHom $(\boldsymbol{A}, \boldsymbol{B})$ the number of transformations preserves the system state from state $\boldsymbol{A}$ to state $\boldsymbol{B}$. The categorical approach only allowed us to formalize the notion of "system", "transformation" and show the invariance of the number of identical structures.

Another methodological remark. Categorical explication allows us to describe systems with different kinds of structures, sometimes very far from the structures of numerical sets. Versatility is used in our paper submission (main functor) in the numerical structure of sets lies in the very design of categories and substantiates the fact of distribution and universal description of various systems numerical characteristics. Indeed, such a representation in a pivotal structure sets exist for any category. Simultaneously become visible the extent to which the existence of a quantitative description of these boundaries warns against the fetishization of numbers in the mathematization of knowledge. The numbers represent the "true" structure only at a certain ratio between the transformations that we have selected for comparison systems of states, and a monomorphism categories. Thus, to compare the number of structures (explication categories) can always be, but not necessarily, these numbers are arranged in the same way as structures. Note that the transition to the invariants for the analysis of structure ordering is not mandatory, but merely a convenient method because of the technique with numerical structure is more developed than arbitrary. "Not cardinal" structure, that is, those ordering which is not consistent with the ordering of cardinal numbers are available direct investigation category methods, but are not subject to the technique of analysis of numerical invariants.

\subsubsection{Extreme principle}

In mathematics and physics, the idea of "optimal action" in nature was embodied in the development of extreme principles. Historically, the first such principle was "Fermat's principle" in geometrical optics. Farm suggested that the light beam chooses not the shortest path as the path of least time. So extreme was formulated first principle remains firm to this day. All geometrical optics is in this principle as a plant in the grain and can be obtained from it by methodical deployment of context-specific traffic light. In this example we can see two main features common to all the principles of extreme: extreme conciseness and simplicity and general and universal. Knowing Fermat's principle, it is possible to calculate any optical system without requiring any other laws of geometrical optics - they are only a consequence of this principle.

Thus, the content of any extreme principle is a statement of the minimum (and maximum) of some magnitude. Reckoning is seen as the search for the "true" path among all the many possible. True motion is different from all the capabilities of its "optimal", that is, the fact that for a certain value reaches a minimum (or maximum). Thus, the main problem is to find the minimized value, and find out its structure. This is not always so easy to do, as in optics, or mechanics.

\subsubsection{Basic Concepts and Elements of the Theory of Fuzzy Sets and Fuzzy Logic}

Present the basic definitions of the operations undertaken in the theory of fuzzy sets, as well as the description and definition of the operations proposed recently in [8].

Under the term plurality understand the totality of elements having a common property. In this case, any item in advance axiomatically belongs to this set or does not belong. However, as the practice of applied research, such a "logical" principle in most cases does not correspond to processes occurring in the real complex systems, and leads to unjustified idealization of the mathematical description of such systems. In other words, it sets the ordinary is not enough flexibility for the formalization of the uncertainties inherent in real systems.

The concept of fuzzy set based on the assumption that every element to some extent belongs to this set, so one of the main ways the mathematical description of a fuzzy set is the definition of a certain number of accessories, for example, in the range [0,1]. The boundaries of the intervals 1 and 0 respectively mean "belongs" and "not belong." 
Suppose $U$ - a set of elements. Fuzzy set $A \subseteq U$ is the set of pairs $\left(u, \mu_{A}(u)\right)$, where $u \in U: \mu_{A}(u): U \rightarrow[0,1]$ (later $\mu_{A}(u): U \rightarrow L$ - the structure of the lattice type) function, which is called the membership function.

Conventional sets constitute a subclass of fuzzy sets, that is the usual membership function sets $B \subset U$ is the characteristic function

$$
\mu_{B}(u)=\left\{\begin{array}{lll}
1, & \text { if } & u \in B ; \\
0, & \text { if } & u \notin B .
\end{array}\right.
$$

A fuzzy set is called blank if its membership function is zero on the entire set $U$ :

$$
\mu_{\varnothing}(u)=0, \quad \forall u \in U .
$$

$U$ universal set can be described by the membership function of the form

$$
\mu_{U}(u)=1, \quad \forall u \in U .
$$

Carrier fuzzy set $\boldsymbol{A}(\sup \boldsymbol{A})$ with membership function $\mu_{A}(u)$ is the set of the form

$$
\sup A=\left\{u \mid \mathrm{u} \in \mathrm{U}, \mu_{A}(u)>0\right\} .
$$

Fuzzy set $\boldsymbol{A}$ is called normal if the equality sup $\mu_{A}(u)=1$, upper limit of the membership function is $u \in U$

equal to 1 .

Otherwise, the fuzzy set called subnormal. Singleton fuzzy called fuzzy set whose support is the only point of the set of $U$, any fuzzy set $\boldsymbol{A}$ can be regarded as the union of its constituent point sets Singleton, which finds expression in the general case

$$
A=\int_{U} \mu_{A}(u) d u, \quad \forall u \in U .
$$

2.1.3. The formulation of the variability law which selects the real generalized motion of objects in the space of all possible states

Considering the universal properties of complex mechanical systems, the processes of self-organization and management of such systems, exploring new qualities of such systems, we use an interdisciplinary approach, which is actively developing - synergy. In this regard, several paradigms justify having scientific importance. Methodology of science today emit three major paradigms of particular importance for the understanding of the unity of scientific knowledge - a systematic approach, the general theory of information and the new concept of self-organization that emerged within the synergy - the paradigm of complexity, which were among the applications and the design of intelligent objects and the creation of a new generation of technology. This micromachines and nanotechnology. Behind them are new materials, the possibility of "molecular growing" complex systems "repair" of individual molecules, a new generation of computer systems. The principal difference between these technologies, and affect the element base, and algorithms, and very tasking. Last paradigm has demonstrated its applicability in various sciences, and although the situation is not unique synergy paradigm for all sciences, it provides a methodological framework and analytical tools for the study of processes in the technology of construction of nanodevices. Since this paradigm describes a "new science", a closer look at it.

In the electronics industry production of substrates is based on machined bulk single crystals (ingots). Ingots are divided into thin sections, so-called plates. To achieve the desired accuracy and surface quality of the plate is subjected to grinding, polishing and chemical treatment. Such a method of production of substrates of integrated circuits (ICs) allows to obtain the required accuracy and surface properties required, but it is associated with a significant quantity of waste of expensive materials. Physical processes associated with the operation of semiconductors (SC) and ICs, occurring in a single crystal layer depth of 15 - 20 microns. The rest of the silicon provides the mechanical strength of the device and directs the heat generated during its operation. Since the finished chip is mounted on a crystal holder, a significant portion of the thickness of silicon could be decreased. Thus, the thickness of the substrates is determined by the strength and technological capabilities. Grinding and polishing operations can not significantly change the macro geometry of the substrate. It is on the cutting operation through the process heredity laid the quality of future substrates.

The analysis shows that for all practical studies arbitrary state, or the state space of a dynamical system, it is possible to identify with a set endowed with a certain mathematical structure. For example, the state of the cutting process of single crystals of the plates is convenient to describe the set of broken into disjoint classes, where the elements of the sets are the elements of cutting schemes that create the cutting process, and the class of the partition - functionality cutting schemes. In the future, the basic provisions of the general theory illustrate the example of the cutting technology of single crystals.

Aspect 1. We use the general systems theory, with its logic, formal languages and ways of rational methodological basis $[3,4]$ for the construction of a mathematical model. We emphasize that the development of any system (in our case the material) - the process technology. If the technology as a way of processing allows the construction of different machinery or processes, then the formal construction of technological algorithms (models) allows you to reveal the basic laws of technology, prerequisites and possible outcomes. Use the ideas set out in [2].

We introduce a definition. Suppose $U$ - a set of elements. $A \subseteq U$ fuzzy set is the set of pairs of the form $\left(u, \mu_{A}(u)\right)$, where $\left(u \in U: \mu_{A}(u): U \rightarrow[0,1]\right)$ - function, which is called the membership function: 


$$
\mu_{A}(u)= \begin{cases}1, & u \in A \\ 0, & u \notin A\end{cases}
$$

The set $A$ - set of some primary objects of nature (eg, assembly units of a node of the mechanical system) is called the basic elements. $\mathbf{R}$ - the set of operations on basic elements. Membership function $\mu_{A}(u)$ allows the best way to structure are not exact status of basic elements and/or operations with them.

Let us assume that the simulated technological process determined by a combination of fuzzy sets $P(U)=\left\{X_{1}, \ldots, X_{n}\right\} \quad$ in $U$ and the set of fuzzy sets $P(V)=\left\{Y_{1}, \ldots, Y_{n}\right\} \quad$ in $V$, while $X_{i}=\left\{\left(x, \mu_{x_{i}}(x)\right)\right\}$; wherein $U$ and $V$ - the input and output end of the object space simulation.

The above technological process will present a fuzzy equation

$$
Y_{i}=Y_{i} \circ \mathbf{R}, \quad i=\overline{1, n},
$$

or

$$
\mu_{i}(y)=\max _{x} \min \left\{\mu_{X_{i}}(x), \mu_{R}(x, y)\right\},
$$

where $\mathbf{R}$ - the matrix of fuzzy relations with elements of $r_{i j}, \quad i=\overline{1, n} ; \quad j=\overline{1, m} ;\left(r_{i j}\right.$ - number in the range [0,1], characterizing the degree of influence of $i$ - the second reason for the occurrence of $j$ - of the consequences) on $U \times V$; operation $\max$-min composition.

At the initial stage will make a choice of elementary objects, and methods of their variability, or structural principles.

Aspect 2. We expand the principle of optimality and generalization - extreme principle. We first note that, in theory, based on the extreme principle, the postulation of a function or functional extremum search method which takes the calculus of variations, leads to a description of the development of technology as a dynamical system under study.

The model describes the choice of technology on non-renewable resource stocks. The model can serve as a prototype of some dynamic systems, such as systems technology selection scheme cutting, and then adaptively selective cutting machine cutting discs on a single crystal wafer. Then a non-renewable resource stocks appears as the value of the deformation of the cutting edge. Given that the choice of technology depends not only on the value of the deformation of the cutting edge, but also the thickness of the plate deflection, vibration level et al., builds a mathematical model:

- technological process simulated cutting machine schemes, where circuits are distinguished according to needs of continuous resource (they can be divided into groups of influence of deformation of the cutting edge changes, the tension of sharpening, the cutting force). Convenient to describe this technological process with a variety of elements $\boldsymbol{n}$ (schemes), broken into of nonintersecting classes of $\boldsymbol{w}$ (by type of movements and types of provisions of the cutting tool and cut a single crystal) with the number of elements in the class $n_{i}\left(i=\overline{1, \mathrm{w}} ; \quad \sum_{i=1}^{w} n_{i}=n\right)$, in other words the mathematical structure a partitioned set with. Classes meet partitioning schemes cutting. Set the number of classifications of movement and position $\vec{n}=\left\{n_{1}, n_{2}, \ldots, n_{w}\right\}$ call state of the system.

Postulate extreme principle.

Postulate 1. The technological process as a dynamic system with a given state to a state of extreme defined structure and allowable by type of movements and positions of the cutting tool in the system. It is necessary to provide the final steady state system in which the dried headroom (the tension of the cutting tool, grinding and others.).

Result: The system is in a state of the most extreme structure to the extent permitted resources.

Model of the process is characterized by a variety of possible choices of her condition. This characteristic may be called entropy models. Usually, the mathematical model allows several definitions of the state, and each definition to answer its own measure of entropy. Meaningful state - a possible mode or method of use of the model. By following the Boltzmann entropy is defined as the logarithm of the number of states of the model, and that is how the entropy measure of the model depends on the definition of the state. We define the entropy as a logarithmic measure $H(\vec{n})$ of certain states for the model $N[9,10]$ :

$$
H(\vec{n})=\log N .
$$

Search real states of the system, among all possible in this methodology requires an ordered state with each other, and a choice of extreme states in the resulting ordering. Let us call this search principle extreme structure. Hence, there is a numerical function of the state - the number of admissible transformations. The number of valid system structure transformation depends on two characteristics of structured sets: the number of elements in it, and on the set of a given structure. Instead, the total number of admissible transformations is necessary to use the specific number - the number per one element of the set of all transformations. The reciprocal of the specific invariant is equal to the number of non-equivalent transformations.

In this case, the specific structure of the invariant sets with the partition is defined as

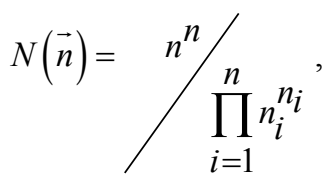

then (3) can be rewritten in the form of generalized entropy 


$$
H(\vec{n})=\ln N(\vec{n})=-n \sum_{i=1}^{w} \frac{n_{i}}{n} \ln \frac{n_{i}}{n} .
$$

Based on the above stated it formulate Assertion 1: any class of open systems changes and consumes some resource.

Assertion 2: permissible changes to the system are always limited deficit of some resource.

Consequence: in the extreme principle that the law generates turnover extremum is always conditional.

Postulate 1 rewrite in a new form.

Postulate 2. Technological process as a dynamic open system from a given state to a state of extreme structure is defined and allowed by type of movements and positions of the cutting tool in the system, for which the generalized maximum entropy within the limits defined acceptable system resources.

Consequence: absolute maximum entropy leads to a uniform distribution system characteristics (second law of thermodynamics). Conditional extremum entropy for open, but limited resource systems, draws inhomogeneous distribution. An arbitrary degree of heterogeneity depends on the components of the system under study.

The requirement for maximum generalized entropy with limited headroom requirement is equivalent to a minimum consumption of these resources for the system of restrictions granularity of the system must not be below a certain threshold $[9,10]$.

Postulate 3. Technological process as a dynamic open system from a given state to a state of extreme structure is defined and allowed by type of movements and positions of the cutting tool in the system, for which the consumption of resources is limited, it should be a prerequisite for structuring the system.

Aspect 3. The study of open systems has been called the principle of maximum entropy. The main problem in the application of this principle is the lack of clear procedures for comparing the investigated system adequate to its nature entropy functional. Therefore, a common practice when working with the principle of maximum entropy is postulating for our system of some analogue of formula (3), for example, the formula (4). The most consistent this trend manifests itself in the formalism of Janes [11], namely:

- compare the valid states of the system (technology or methods of processing) certain probabilities (or inaccuracy) of their implementation;

- formulate the equations in the form of restrictions on the macro parameters of the system (for example, the measurement accuracy deviation of the actual dimensions of parts to be adaptive - selective assembly);

- find an equilibrium state of the system (technology or processing method) method of Lagrange multipliers as the solution of a conditional with a maximum of functionality in the form of, for example, according to Shannon entropy.

Together with a justification of the entropy functional it is important to solve the problem of selecting adequate restrictions on its extremum because before solutions the variational problem is not known to what settings should be made in the form of restrictions to strict rules.

We formulate the variational problem on a conditional extremum (Postulate 2):

$$
\left.\begin{array}{cc}
H(\vec{n})=\left(\sum_{i=1}^{w} n_{i}\right) \ln \left(\sum_{i-1}^{w} n_{i}\right)-\sum_{i=1}^{w} n_{i} \ln n_{i} & \rightarrow \max ; \\
\sum_{i=1}^{w} q_{i}^{k} n_{i} \leq L^{k}, & k=\overline{1, m} \\
n_{i} \geq 0 ; & i=\overline{1, w}
\end{array}\right\}
$$

where $\boldsymbol{n}_{\boldsymbol{i}}$ - end circuit cutting each disjoint classes $\boldsymbol{i}$, which must be found; $\boldsymbol{q}_{i}{ }^{\boldsymbol{k}}$ - number $\boldsymbol{k}$ - th resource disjoint classes $\boldsymbol{i}$; $\boldsymbol{m}$ - the total amount of resources; $\boldsymbol{w}$ - the total number of classes of circuits cutting; $\boldsymbol{L}^{\boldsymbol{k}}$ - the initial value of the resource $\boldsymbol{k}$.

$$
\begin{gathered}
\text { The solution of } \quad \text { (5) for any vector } \\
\vec{L} \in \mathbf{R}^{m}=\left\{\vec{L} \in \mathbf{R}^{m} \mid \vec{L}>0, k=\overline{1, m}\right\} \\
n_{i}=n \exp \left(-\vec{\lambda}, \overrightarrow{q_{i}}\right), \quad i=\overline{1, m}
\end{gathered}
$$

where $\overrightarrow{q_{i}}=\left(q_{i}^{1}, q_{i}^{2}, \ldots, q_{i}^{m}\right)$ - number of allowable by type of movements and positions of the cutting tool in the system (resource), and the total number of schemes cutting $n=\sum_{i=1}^{w} n_{i}$ and vector $\vec{\lambda}=\left(\lambda^{1}, \ldots, \lambda^{m}\right)$ is a solution of algebraic equations

$$
\left.\begin{array}{cc}
\sum_{i=1}^{w} \exp \left(-\vec{\lambda}, \overrightarrow{q_{i}}\right)=1 ; & \\
\lambda^{k}\left(n \sum_{i=1}^{w} q_{i}^{k} \exp \left(-\vec{\lambda}, \overrightarrow{q_{i}}\right)-L^{k}\right)=0, & k=\overrightarrow{1, m} ; . \\
\lambda^{k}>0, & k=\overline{1, m}
\end{array}\right\}
$$

\subsection{The Presentation Layer of the Studied System}

Note that the theory of categories and functors [6,7] includes methods of comparison in terms of structuring the various states of the simulated systems, where at an abstract level description of the system there is the possibility to calculate quantitative structural characteristics of the states, and with their help to identify extreme state of the system (ie, postulated function or functional extremum search methods of calculus of variations which leads to the description of "formal technology" as the system under study).

If the theory of sets display design is a derivative with 
respect to themselves and auxiliary sets [3], in category theory [6,7] conversion facilities are included in the axiomatic definition of the category on a par with objects. Objects are selected, the limiting case of transformation. When categorical - functorial description of systems the focus is shifting from the states of the objects in the various forms of movements and transformations. The subject of the study is not only the state of the system, but also how they change.

In all cases of practical interest arbitrary state (state space) open dynamic system fails to identify with a set endowed with a certain mathematical structure. For example, the technology of cutting ingots into wafers is convenient to describe a set with a partition into disjoint classes, where the elements of the circuit elements are cutting and splitting class - technological schemes of cutting, combined by type of movements and types of provisions of the cutting tool and the single crystal.

Thus, in the general case state space of a dynamical system is an open class equally structured sets.

Assume that the $\boldsymbol{A}$ and $\boldsymbol{B}$ - are equally structured sets.

We introduce the notation. Conformity of $c$ is a subset of $c \subset A \times B$.

We denote

$$
\left\{\begin{array}{cc}
c(a) \equiv\{b \mid(a, b) \in c\} \subset B, & a \in A ; \\
c^{-1}(b) \equiv\{a \mid(a, b) \in c\} \subset A, & b \in B .
\end{array}\right.
$$

Possibly, $c(a)=\varnothing$ and $c^{-1}(b)=\varnothing$ in some $\boldsymbol{a}$ and $\boldsymbol{b}$.

We note some types of correspondences with $\boldsymbol{A}$ to $\boldsymbol{B}$ :

$$
\left\{\begin{array}{lll}
p \subset A \times B, & p(a) \neq \varnothing, & a \in A ; \\
s \subset A \times B, & s^{-1}(b) \neq \varnothing, & b \in B ; \\
f \subset A \times B, & f(a) \neq \varnothing, & a \in A ; \\
i \subset A \times B, & i^{-1}(b) \neq \varnothing, & b \in B .
\end{array}\right.
$$

The first conformity is called a defined everywhere; the second - surjective; the following functionality for which $f(a)$ is either empty or consists of a single element; and last - injective for which $i^{-1}(b)$ is either empty or consists of one element. However, different combinations of these are valid conformity. For example, certain functionality and conformity are identified throughout with conventional reflections. Conformity that preserve the mathematical structure defined on the set $\boldsymbol{A}$ and $\boldsymbol{B}$, called a morphism structure and denoted $\alpha, \beta, \gamma, \ldots$.

Based on the foregoing, the transitions between states of arbitrary admissible open dynamic system are identified with the morphism of the corresponding mathematical structure. In addition, each system is characterized by a specific, peculiar only to her class of morphisms.

So, with all kinds of open dynamical system is supposed to link the mathematical structure $S$, consisting of two classes: $\mathrm{ObS}$ and MorS:

$\boldsymbol{O b S}$ - fixed class of are equally structured sets;
MorS - fixed class structure morphisms given on these sets.

We note some properties of the mathematical model:

1. Each ordered pair of objects $(A, B) \in O b S \times O b S$ corresponds to a set $\operatorname{Mor}_{S}(A, B) \subset M o r S$ structure morphisms from $\boldsymbol{A}$ to $\boldsymbol{B}$. $\operatorname{Mor}_{S}(A, B)=\varnothing$ for some $\boldsymbol{A}$ and $\boldsymbol{B}$;

2. Every morphism of class $\boldsymbol{M o r} S$ belongs to one and only one set of $\operatorname{Mor}_{S}(A, B)$ where $A, B \in O b S$;

3. For each triple of objects $A, B, C \in O b S$ in class Mor $S$ introduced the operation of multiplication of morphisms: $\operatorname{Mor}_{S}(A, B) \times \operatorname{Mor}_{S}(B, C) \rightarrow \operatorname{Mor}_{S}(A, C)$, $(\alpha, \beta) \rightarrow \alpha \beta \quad$, here $\alpha \beta \in \operatorname{Mor}_{S}(A, C) \quad$ - the composition of morphisms $\alpha \in \operatorname{Mor}_{S}(A, B)$ and $\beta \in \operatorname{Mor}_{S}(B, C)$, certain the terms: $(a, c) \in \alpha \beta$ when $a \in A$ and $c \in C$ if and only if there is an element of $b \in B$, such that simultaneously $(a, b) \in \alpha$ and $(b, c) \in \beta$;

4. For each $A \in O b S$ there exists an identity morphism $1_{A} \in \operatorname{Mor}_{S}(A, A)$, such that $1_{A} \alpha=\alpha$ and $\beta 1_{A}=\beta$ in all $\alpha \in \operatorname{Mor}_{S}(A, B), \quad \beta \in \operatorname{Mor}_{S}(C, A) \quad$ and $C, B \in O b S$. This is the identity conformity $1_{A}=\{(a, a) \mid a \in A\} \subset A \times A$.

Thus, $\boldsymbol{S}$ is a category.

Thus, the proposed model complex systems has a simple theoretical - categorical interpretation that allowed the system state is an object, and the allowed transitions between these conditions - morphism.

The next important step in the description of a complex system is to establish the extreme principle that allows you to select from a set of allowable only those states which are realized in the given conditions. To do this, you must construct a function on the state space of the system with values in a linearly ordered set, because only meaningful concept "extreme state". We find that the function defined on the class $\boldsymbol{O b S}$

First of all, let us recall some facts about the cardinal number of unstructured sets. Consider the category Set, whose objects are arbitrary sets and morphisms correspondences between these sets. A binary relation on the class ObSet agree called subclasses direct product ObSet $\times$ ObSet .

Suppose $R_{1}$ - a binary relation on $\mathrm{ObSet}$, defined by the rule: $(A, B) \in R_{1} \Leftrightarrow$ exists an injective mapping from $\boldsymbol{A}$ to $\boldsymbol{B}$. Clearly, $R_{1}$ is reflexive and transitive, and is the ratio of pre-order $\mathrm{ObSet}$. $R_{1}$ factored pre-order by introducing the same class $O b S e t$ binary relation $E_{1}$ according to the rule: $(A, B) \in E_{1} \Leftrightarrow(A, B) \in R_{1}$ and $(B, A) \in R_{1}$. 
Such a definition of the ratio $E_{1}$ is reflexive, transitive and symmetric. Thus, $E_{1}$ is an equivalence relation on ObSet .

For each $A \in O b S$ by $[A]_{E_{1}}$ denote the class of all sets, $E_{1} \quad$ - equivalent to the set $A$, ie put $[A]_{E_{1}}=\left\{A^{\prime} \mid\left(A, A^{\prime}\right) \in E_{1}\right\} \subset$ ObSet . This class is usually called the cardinal number of the set $\boldsymbol{A}$ and write $\operatorname{CardA}=[A]_{E_{1}}$ (if $\boldsymbol{A}$ - finite set, then $\operatorname{CardA}$ is the number of elements in $\boldsymbol{A})$. In other words, the cardinal numbers of unstructured sets are the elements of the factor-class $\mathrm{ObSet} / E_{1}$. We have both canonical surjection $\operatorname{CardA}: \mathrm{ObSet} \rightarrow \mathrm{ObSet} / E_{1}, \mathrm{~A} \rightarrow \operatorname{CardA}$. By a factor of class $\mathrm{ObSet} / E_{1}$ naturally determined by the linear order relation $" \leq_{1} "$.

Extend the scheme described above reasoning to the case of category $\boldsymbol{S}$ equally structured sets. Suppose $A, B \in O b S$. By analogy with the general algebra injective map from $\boldsymbol{A}$ to $\boldsymbol{B}$ (to and from $\boldsymbol{B}$ to $\boldsymbol{A}$ ), keeping the mathematical structure defined on these sets is called monomorphisms structure.

Consider a binary relation $R_{2}$ on the class $O b S$, defined by the following rule: $(A, B) \in R_{2} \Leftrightarrow$ there exists a monomorphism structure with $\boldsymbol{A}$ to $\boldsymbol{B}$. Clearly, the ratio $R_{2}$ is reflexive and transitive, ie, is preorder to $O b S$. Factorization preorder $R_{2}$ standard way generates an equivalence relation on $E_{2}$ the same class $O b S$ : $(A, B) \in E_{2} \Leftrightarrow(A, B) \in R_{2}$ and $(B, A) \in R_{2}$.

For each $A \in O b S$ introduce a class $[A]_{E_{2}}=\left\{A^{\prime} \mid\left(A, A^{\prime}\right) \in E_{2}\right\} \subset O b S e t$ equally structured sets, $E_{2}$ - equivalent to the set $\boldsymbol{A}$. Let us call this class the set $\boldsymbol{A}$ number of structural and use it to refer to: $\operatorname{Str} A=[A]_{E_{2}}$.In this way, number of structural by definition, are the elements of a factor - class $O b S e t / E_{2}$. We have both canonical surjection Str $: O b S \rightarrow O b S / E_{2}, A \rightarrow S t r A$.

With $R_{2}$ we define a factor - class $\mathrm{ObSet} / E_{2}$ order relation, assuming $\operatorname{Str} A \leq_{2} \operatorname{Str} B \Leftrightarrow(A, B) \in R_{2}$, checking the reflection, transitive, and antisymmetric this particular relationship " $\leq_{1} "$. However, unlike the cardinal, structural part number ordered.

Thus, none of the functions on $O b S$, takes the value in a factor- class $\mathrm{ObSet} / \mathrm{E}_{2}$, despite the partial ordering of the latter, can not serve as a satisfactory basis for the extreme principle.

Way out - to seek ways of building submission structural numbers more convenient for our purposes structure of order.
For this purpose we use the fact that $S$ is category and factor-class $\mathrm{ObSet} / E_{1}$ category Set linearly ordered.

We fix a class $O b S$ arbitrary object $\boldsymbol{A}$. Consider the reflection of $F_{A}: S \rightarrow$ Set, comparing each $B \in O b S$ set of $F_{A}(B)=\operatorname{Mor}_{S}(A, B) \in \mathrm{ObSet}$ morphisms structure from $\boldsymbol{A}$ to $\boldsymbol{B}$ and every $\beta \in \operatorname{Mor}_{S}(B, C) \subset M o r S$ everywhere defined functional correspondence $F_{A}(\beta)=\left\{(\alpha, \alpha \beta) \mid \alpha \in \operatorname{Mor}_{S}(A, B)\right\}$ $\subset \operatorname{Mor}_{S}(A, B) \times \operatorname{Mor}_{S}(A, C)$ of $F_{A}(B) \quad$ in $F_{A}(C)$ any $B, C \in O b S$.

Thus, the mapping $F_{A}$ is a Single covariant functor from the category of $\boldsymbol{S}$ in the category of Set [6,7], known as displaying functor.

We say that the cardinal number $J_{A}(B) \equiv \operatorname{CardMor}_{S}(A, B)=\operatorname{CardF}_{A}(B)$ invariant object $B \in O b S$ relative to the object $A \in O b S$. For a fixed $A$ have the function $J_{A}: O b S \rightarrow O b S e t / E_{1}, B \rightarrow J_{A}(B)$. It is easy to see, is the composition of the injection $F_{A}^{O b}$ : $\mathrm{ObS} \rightarrow \mathrm{ObSet}, \quad B \rightarrow F_{A}(B)$ and canonical surjection Card : ObSet $\rightarrow \mathrm{ObSet} / E_{1}$. Moreover, the following assertion: if $\operatorname{Str} B \leq_{2} \operatorname{Str} C$, then $J_{A}(B) \leq_{1} J_{A}(C)$. indeed, $\operatorname{Str} B \leq_{2} \operatorname{Str} C$ condition for means that there exists a monomorphism structures from $\boldsymbol{B}$ to $\boldsymbol{C}$.

Thus, the objects of the category $\boldsymbol{S}$ serve to compare the invariant by comparing them. This fact is at the heart of functor comparison method of structured sets.

Function $J_{A}$ defined on the whole $O b S$ and takes values in a linearly ordered factor - class $\mathrm{ObSet} / \mathrm{E}_{1}$, that is, it may well be used in extreme principle. Bearing in mind the subsequent applications to a limited complex systems, starting from this moment, focusing on finite sets.

Let us now consider as $\boldsymbol{S}$ category $F S_{a}$, such that the class $O_{b F S_{a}}$ consists of finite equally structured sets and class $\operatorname{MorFS}_{a}$ - morphisms of structures with properties matching, multi-index marked " $a$ ". Category $F S_{a}$ already provides sufficient means for modeling a wide range of complex systems.

Suppose $A, B \in O b F S_{a}$ and $J_{A}^{F S_{a}}(B)$ - invariant object $\boldsymbol{B}$ with respect to object $\boldsymbol{A}$ in the category $F S_{a}$, ie $J_{A}^{F S_{a}}(B)=\operatorname{CardMor}_{F S_{a}}(A, B)$. Consider the quantity $J_{A}^{a}\left(B^{\prime}\right) / J_{A}^{F S_{a}}(B)$, known as the specific invariant $B \in O b F S_{a}$ relatively $A \in O b F S_{a}$. Specific invariant is convenient to choose a numerical measure of the distance of structured sets $\boldsymbol{A}$ and $\boldsymbol{B}$ from the respective carriers $A^{\prime}$ and $B^{\prime}$ mathematical structure. Relatively complex systems, 
this means that the state of the system with high values of specific invariants more "structured" than the states with smaller values of the latter. The above makes it appropriate introduction of such a definition:

Entropy $H_{A}^{a}(B)$ state $B \in O b F S_{a}$ regarding the status of $A \in O b F S_{a}$ a complex system is called the logarithm of the corresponding specific invariant, ie, $H_{A}^{a}(B)=\ln \frac{J_{A}^{a}\left(B^{\prime}\right)}{J_{A}^{F S_{a}}(B)}$. For a fixed $\boldsymbol{A}$ entropy $H_{A}^{a}(B)$, obviously, is a function of $B \in O b F S_{a}$ with values in semi $[0,+\infty)$. If classes states between which transitions are allowed, for example, because of the conservation system macroparameters interpreted as macrostate of the system, and the result is an arbitrary transformation system interpreted as its microstate, the value $H_{A}^{a}(B)$ can be regarded as a generalization of Boltzmann's entropy, is usually defined as the logarithm of the specific number of microstates than its corresponding macroparameters. The possibility of this interpretation and the coincidence of individual cases with a value of $H_{A}^{a}(B)$ traditional formulas for Boltzmann's entropy were the basis for the use of the term "entropy". Note that in the proposed context, the term entropy is there any considerations of probability.

Now it is clear how to formulate the principle of extreme managing the behavior of the complex system. Adopt such a postulate:

- from a given state of the system $A \in O b F S_{a}$ in reality a transition to the state $B \in O b F S_{a}$ for which the entropy of the $H_{A}^{a}(B)$ maximum within permitted by external conditions (eg available energy and other resources).

\section{Conclusions}

3.1. A model of process control complex technical systems based on the mathematical theory of categories and functors.

The system state (or solution curve of the system) can be arranged (determined) by the number of admissible transformations.

Generalized entropy state of the system (on the solution curve) can be determined by the logarithm of the number of admissible transformations and interpreted as a measure of optimality.

For properties of control systems, such as the controllability, stability optimality solutions can be proposed formalism based on maximum entropy.

3.2. Shows original data to develop a model of fuzzy inference to control the cutting process of the single crystal silicon, which can be used in the stabilization system parameters of the system. Such a system would minimize the negative impact, and to solve the problem of formation of the required parameters on the output while reducing the computational cost.

Proposed preconditions to optimal control algorithm based on fuzzy inference system, when the basis for the operation of fuzzy inference is a rule base containing fuzzy statements in the form of "If, Then" and the membership functions for the respective linguistic terms. At the same time the following conditions: there is at least one rule for each linguistic term output variable. For any term of the input variable has at least one rule in which this term is used as the preconditions (left side of the rule). Otherwise, there is a part-time basis of fuzzy rules.

In general, the inference engine includes four stages: introduction of fuzziness (Fuzzification), fuzzy inference, composition and leading to clarity, or defuzzification. Thus, the fuzzy inference algorithms differ mainly in view of the rules used, logical operations and a variety of defuzzification method.

\section{REFERENCES}

[1] Ludwig von Bertalanffy. General system theory: foundations, development, applications. G. Braziller, New York, 1968.

[2] M.D.Mesarovic and Y.Takahara, General systems theory: Mathematical foundations. Mathematics in Science and Engineering, Vol. 113, Academic Press, New York, 1975.

[3] N. Bourbaki. Theory of Sets. Springer Science \& Business Media, 2004. This is a softcover reprint of the English translation of 1968 of N. Bourbaki's, Théorie des Ensembles (1970).

[4] N.Bourbaki. The Architecture of Mathematics. The American Mathematical Monthly, Vol. 57, No.4. (Apr., 1950), pp. 221-232.

[5] N.Bourbaki. Algebra I: Chapters 1-3. Springer Science \& Business Media, 1998. This softcover reprint of the 1974 English translation of the first three chapters of Bourbaki's Algebre gives a thorough exposition of the fundamentals of general, linear, and multilinear algebra.

[6] I.Bucur, A.Deleanu. Introduction to the Theory of categories and functors. A Wiley-Interscience Publication John Wiley \& Sons, LTD. London New York - Sydney, 1968.

[7] R.Goldblatt. Topoi. The categorical analysis of logic. Dover Publications, INC. Mineola, New York, 2006.

[8] Fundamentals of Fuzzy Sets. Edited by Didier Dubois and Henri Prade; foreword by Lotfi A.Zadeh. The handbooks of fuzzy sets series; FSHS 7. Springer Science \& Business Media, 2000

[9] Henryk Gzyl. The method of maximum Entropy. (Series on Advances in Mathematics for Applied Sciences). Singapore, New Jersey, London, Hong Kong: World Scientific. 1995.

[10] Henryk Gzyl, Enrique ter Horst. A Relationship between the Ordinary Maximum Entropy Method and the Method of Maximum Entropy in the Mean. Entropy 2014, 16, 1123-1133.

[11] Janes E.T. Information theory and statistical mechanics I. Phys. Rev. 1957. V.106. №4. Pp. 620-630. II. Phys. Rev. 1957. V.108. №2. 171-190. 